\title{
Monocyte High Density Lipoprotein Cholesterol Ratio as a New Prognostic Factor for Mesenteric Embolism
}

\author{
Adnan Kuvvetli ${ }^{1}$ and Begum Seyda Avci ${ }^{2}$ \\ ${ }^{1}$ Department of General Surgery, Health Science University, Adana City Research and Training Hospital, Turkey \\ ${ }^{2}$ Department of Internal Medicine, Health Science University, Adana City Research and Training Hospital, Adana, Turkey
}

\begin{abstract}
Objective: To determine the effectiveness of the ratio of monocyte count to high density lipoprotein cholesterol in predicting in-hospital mortality.

Study Design: Descriptive study.

Place and Duration of Study: Intensive Care Unite, Adana Numune Research and Training Hospital, Turkey, from January 2018 to December 2019.

Methodology: Patients admitted to the intensive care unit with the diagnosis of mesenteric embolism were included in the study. Monocyte count and high-density lipoprotein cholesterol (HDL) values were determined. Monocyte HDL Ratio (MHR) values of the patients were calculated. SPSS 26 package programme was used to investigate the effectiveness of MHR in predicting mortality.

Results: The mean age of the 81 patients was $69.9 \pm 10.6$ years. In the group with mortality, the number of monocytes and MHR were significantly higher than the group without mortality $(p<0.05)$. In the mortality group, HDL value was significantly lower $(p<0.05)$ than the non-mortality group. Sensitivity of the MHR cutoff value of 19 was $81.8 \%$, positive predictive value was $96.4 \%$, specificity value was $97.9 \%$, negative predictive value was $88.7 \%$.

Conclusion: For patients diagnosed with acute mesenteric embolism, the use of predictors in terms of mortality estimation is very important for the faster implementation of treatment modalities. MHR value can be used as a strong predictor.
\end{abstract}

Key Words: Mesenteric embolism, Monocyte, HDL cholesterol, MHR, Mortality.

How to cite this article: Kuvvetli A, Avci BS. Monocyte High Density Lipoprotein Cholesterol Ratio as a New Prognostic Factor for Mesenteric Embolism. J Coll Physicians Surg Pak 2020; 30(07):688-693.

\section{INTRODUCTION}

Acute mesenteric embolism is a disease with a high mortality rate that can cause acute abdominal pain. Despite recent developments in diagnosis and treatment methods, the mortality rate of this disease is approximately $40-70 \% .{ }^{1}$ Serum markers have been investigated as easy-to-apply methods of assisting in early diagnosis of acute mesenteric embolism. However, the role of serum markers in the diagnosis of this disease is quite limited.

Previous studies have shown that an increase in the number of monocytes and a decrease in HDL levels may be associated with inflammation and oxidative stress. ${ }^{2-5}$ Many studies have used MHR to determine whether inflammation and atherosclerosis contribute to the etiopathogenesis of cardiovascular and cerebrovasculardiseases. ${ }^{6-11}$

Correspondence to: Begum Seyda Avci, Department of Internal Medicine, Health Science University, Adana City Research and Training Hospital, Turkey

E-mail: begumtngnr@hotmail.com

Received: February 11, 2020; Revised: June 08, 2020;

Accepted: July 14, 2020

DOI: https://doi.org/10.29271/jcpsp.2020.07.688
The results of these studies emphasised that MHR is an independent predictor in relation to inflammation caused by vascular injury.

Although many studies have examined the relationship between MHR and inflammatory processes, no research has examined the relationship between mesenteric embolism and MHR to the best of authors' knowledge.

The aim of the present study was to determine whether MHR is effective in predicting the in-hospital mortality of patients diagnosed with mesenteric embolism.

\section{METHODOLOGY}

Patients hospitalised in the intensive care unit of Adana Numune Research and Training Hospital, Turkey with diagnoses of mesenteric embolism from January 2018 to December 2019 were scanned from the medical information system. This process yielded the files of 90 patients diagnosed with mesenteric embolism, according to the international diagnostic code. The criteria for inclusion were age over 18 years, full data access, radiological diagnosis of mesenteric embolism in the superior and/or inferior mesenteric artery, not refusing treatment, having been admitted to the study centre, and presenting within first four hours of symptoms initiation. 
Table I: Evaluation of patients' demographic, hematological and biochemical parameters.

\begin{tabular}{|c|c|c|c|c|c|c|c|}
\hline \multirow[b]{2}{*}{ Age } & \multicolumn{3}{|c|}{ Min-Max } & \multirow{2}{*}{$\begin{array}{c}\text { Median } \\
71\end{array}$} & \multicolumn{3}{|c|}{ Mean \pm SD./n-\% } \\
\hline & 53 & - & 87 & & 69.96 & \pm & 10.58 \\
\hline $\begin{array}{l}\text { Gender: } \\
\text { Male } \\
\text { Female }\end{array}$ & & & & & $\begin{array}{l}39 \\
42\end{array}$ & & $\begin{array}{l}48.1 \\
51.9\end{array}$ \\
\hline Fever $\left({ }^{\circ} \mathrm{C}\right)$ & 36.2 & - & 37.2 & 36.5 & 36.50 & \pm & 0.27 \\
\hline Heart rate (pulse/minute) & 64 & - & 150 & 91 & 93.8 & \pm & 16.2 \\
\hline $\mathrm{SBP}(\mathrm{mm}-\mathrm{Hg})$ & 80 & - & 162 & 120 & 118.90 & \pm & 17.26 \\
\hline $\mathrm{DBP}(\mathrm{mm}-\mathrm{Hg})$ & 50 & - & 99 & 70 & 69.09 & \pm & 9.59 \\
\hline Glucose (mg/dL) & 56 & - & 248 & 136 & 143.02 & \pm & 43.73 \\
\hline Urea (mg/dL) & 13.9 & - & 86.1 & 40.5 & 42.07 & \pm & 14.96 \\
\hline Creatinin (mg/dL) & 0.31 & - & 1.64 & 0.99 & 1.00 & \pm & 0.35 \\
\hline $\mathrm{ALT}(\mathrm{U} / \mathrm{L})$ & 11 & - & 47 & 21 & 24.01 & \pm & 10.88 \\
\hline AST (U/L) & 12 & - & 49 & 31 & 30.32 & \pm & 10.37 \\
\hline Sodium (mEq/L) & 134 & - & 149 & 138 & 138.44 & \pm & 3.19 \\
\hline Potassium (mEq/L) & 3.43 & - & 5.32 & 4.12 & 4.22 & \pm & 0.55 \\
\hline Calcium (mg/dL) & 7.1 & - & 9.8 & 8.6 & 8.5 & \pm & 0.8 \\
\hline Albumine (g/L) & 33.1 & - & 47.6 & 37.2 & 37.86 & \pm & 2.55 \\
\hline Hemoglobin (g/dL) & 10.1 & - & 16.9 & 12.3 & 12.45 & \pm & 1.44 \\
\hline Hematocrite (\%) & 30.7 & - & 48.3 & 37.1 & 37.42 & \pm & 4.56 \\
\hline Platelets $\left(10^{3} / \mu \mathrm{l}\right)$ & 109 & - & 760 & 252 & 273.54 & \pm & 117.24 \\
\hline Monocyte $(/ \mu \mathrm{l})$ & 580 & - & 1254 & 812 & 836.85 & \pm & 174.25 \\
\hline $\mathrm{HDL}(\mathrm{mg} / \mathrm{dL})$ & 33 & - & 56 & 46 & 45.77 & \pm & 4.68 \\
\hline MHR & 10.74 & - & 31.62 & 17.91 & 18.54 & \pm & 4.67 \\
\hline $\begin{array}{l}\text { Surgery: } \\
\text { No } \\
\text { Yes }\end{array}$ & & & & & $\begin{array}{l}10 \\
71 \\
\end{array}$ & & $\begin{array}{l}12.3 \\
87.7\end{array}$ \\
\hline Discharge & & & & & 48 & & 59.3 \\
\hline Death & & & & & 33 & & 40.7 \\
\hline
\end{tabular}

A total of 81 patients met these criteria and were included in the study. Patients who were under the age of 18 years, had missing data or whose data could not be accessed, left the hospital by refusing treatment, had non-occlusive mesenteric ischemia in radiological reporting, and patients who died before the diagnosis of mesenteric embolism were excluded. Patients' age, gender, abdominal computed tomography reports, whether they had an operation or not, hospitalisation times were recorded. The diagnosis of mesenteric embolism was made on the basis of reported findings on contrast enhanced computed tomography.

According to the guidelines of the European Society for Trauma and Emergency Surgery, medical treatments included fluid replacement with crystalloids, standard unfractioned heparin infusion, and broad-spectrum intravenous antibiotic therapy. ${ }^{12}$ All participants were admitted to the intensive care unit. At follow-up, laparotomy was applied to patients who presented with evidence of peritonitis or developed later and perforation was detected in direct abdominal X-ray. Necrotic bowel resection and embolectomy were performed in patients undergoing laparotomy. These patients were also given standard anticoagulant therapy.

Blood samples were taken at the time of admission to the emergency medicine department and within the first hours of symptom development. Hemogram and biochemical parameter measurements were taken on venous blood samples from the antecubital region at the time of application. WBC count, hemoglobin, hematocrit, and monocyte values were determined and recorded from the hemogram parameters. Glucose, urea, creatinine, aspartate aminotransferase (ALT), alanine aminotransferase (AST), sodium, potassium, calcium, albumin, and HDL cholesterol values were determined and recorded from the biochemistry parameters. The MHR of each sample was obtained by dividing the number of monocytes $(/ \mu \mathrm{l})$ by the HDL cholesterol (mg/dL) level. Complete blood count measurements were taken using the Sysmex XN 10 (Automated Hematology Analyzer XN series, Sysmex Corporation, 1-5-1 WakinnohamaKaigandori Chuo-ku, Kobe 651-0073, Japan) automated measuring device. 
Table II: Comparison of vital sings, hematological and biochemical parameters of groups.

\begin{tabular}{|c|c|c|c|c|c|}
\hline & \multicolumn{2}{|c|}{ Discharge } & \multirow{2}{*}{\multicolumn{2}{|c|}{$\begin{array}{c}\text { Exitus } \\
\text { Median (IQR) / Mean } \pm \text { S.D }\end{array}$}} & \multirow{2}{*}{ p-value } \\
\hline & \multicolumn{2}{|c|}{ Median (IQR) / Mean \pm S.D } & & & \\
\hline Age & \multicolumn{2}{|c|}{$69.0 / 60.0-80.8$} & \multicolumn{2}{|c|}{$72.0 / 63 .-79.0$} & $0.561^{\mathrm{m}}$ \\
\hline $\begin{array}{l}\text { Gender: } \\
\text { Male } \\
\text { Female }\end{array}$ & $\begin{array}{l}27(n) \\
21(n)\end{array}$ & $\begin{array}{l}56.3(\%) \\
43.8(\%)\end{array}$ & $\begin{array}{l}12(n) \\
21(n)\end{array}$ & $\begin{array}{l}36.3(\%) \\
63.7(\%)\end{array}$ & $0.078^{x^{2}}$ \\
\hline Fever $\left({ }^{\circ} \mathrm{C}\right)$ & \multicolumn{2}{|c|}{$36.5 / 36.2-36.7$} & \multicolumn{2}{|c|}{$36.4 / 36.2-36.7$} & $0.482^{m}$ \\
\hline Heart rate (pulse/minute) & \multicolumn{2}{|c|}{ 89.0/78.0-101.5 } & \multicolumn{2}{|c|}{$98.0 / 88.0-106.0$} & $0.088^{m}$ \\
\hline $\mathrm{SBP}(\mathrm{mm}-\mathrm{Hg})$ & \multicolumn{2}{|c|}{$120 / 110.0-134.8$} & \multicolumn{2}{|c|}{$114.0 / 98.0-130.0$} & $0.057^{\mathrm{m}}$ \\
\hline $\mathrm{DBP}(\mathrm{mm}-\mathrm{Hg})$ & \multicolumn{2}{|c|}{$70.0 / 60.5-75.0$} & \multicolumn{2}{|c|}{$69.0 / 60.0-76.0$} & $0.533^{m}$ \\
\hline Glucose (mg/dL) & \multicolumn{2}{|c|}{$138.5 / 108.0-172.8$} & \multicolumn{2}{|c|}{ 128.0/109.5-185.5 } & $0.382^{\mathrm{m}}$ \\
\hline Urea (mg/dL) & \multicolumn{2}{|c|}{$40.8 \pm 15.6$} & \multicolumn{2}{|c|}{$44.0 \pm 14.0$} & $0.348^{t}$ \\
\hline Creatinin (mg/dL) & \multicolumn{2}{|c|}{$0.8 / 0.7-1.3$} & \multicolumn{2}{|c|}{$1.2 / 0.8-1.4$} & $0.119^{\mathrm{m}}$ \\
\hline ALT (U/L) & \multicolumn{2}{|c|}{$18.0 / 15.0-26.0$} & \multicolumn{2}{|c|}{$24.0 / 17.0-42.0$} & $0.050^{\mathrm{m}}$ \\
\hline AST (U/L) & \multicolumn{2}{|c|}{$25.5 / 21.3-38.0$} & \multicolumn{2}{|c|}{$36.0 / 23.0-42.0$} & $0.068^{\mathrm{m}}$ \\
\hline Sodium (mEq/L) & \multicolumn{2}{|c|}{$137 / 136.0-140.0$} & \multicolumn{2}{|c|}{$139.0 / 136.0-144.0$} & $0.108^{\mathrm{m}}$ \\
\hline Potassium (mEq/L) & \multicolumn{2}{|c|}{$4.0 / 3.8-4.5$} & \multicolumn{2}{|c|}{$4.1 / 3.6-5.0$} & $0.495^{m}$ \\
\hline Calcium (mg/dL) & \multicolumn{2}{|c|}{ 8.7/7.8-9.3 } & \multicolumn{2}{|c|}{ 8.5/7.8-9.2 } & $0.510^{\mathrm{m}}$ \\
\hline Albumine (g/L) & \multicolumn{2}{|c|}{$38.0 / 35.8-39.7$} & \multicolumn{2}{|c|}{$36.9 / 36.2-38.8$} & $0.322^{m}$ \\
\hline Hemoglobin (g/dL) & \multicolumn{2}{|c|}{$12.3 / 11.1-14.0$} & & 3.0 & $0.855^{\mathrm{m}}$ \\
\hline Hematocrite (\%) & & & & & $0.875^{t}$ \\
\hline Platelets $\left(10^{3} / \mu \mathrm{l}\right)$ & 25 & 05.5 & 258 & 349.0 & $0.571^{m}$ \\
\hline Monocyte $(/ \mu \mathrm{l})$ & 71 & 95.3 & 1005 & 1093.5 & $<0.001^{m}$ \\
\hline $\mathrm{HDL}(\mathrm{mg} / \mathrm{dL})$ & & & & 7.5 & $<0.001^{m}$ \\
\hline MHR & & & & 5.1 & $<0.001^{m}$ \\
\hline $\begin{array}{l}\text { Surgery: } \\
\text { No } \\
\text { Yes }\end{array}$ & $\begin{array}{c}4(n) \\
44(n)\end{array}$ & $\begin{array}{c}8.3(\%) \\
91.7(\%)\end{array}$ & $\begin{array}{c}6(n) \\
27(n)\end{array}$ & $\begin{array}{l}18.2(\%) \\
81.8(\%)\end{array}$ & $0.302^{x^{2}}$ \\
\hline
\end{tabular}

Table III: Logistic regression analysis for monocyte, HDL and MHR.

\begin{tabular}{|c|c|c|c|c|c|c|c|c|c|c|}
\hline \multirow[b]{3}{*}{ Monocyte } & \multicolumn{5}{|c|}{ Univariate model } & \multicolumn{5}{|c|}{ Multivariate model } \\
\hline & \multirow{2}{*}{$\begin{array}{c}\text { OR } \\
1.018 \\
\end{array}$} & \multicolumn{3}{|c|}{$95 \% \mathrm{Cl}$} & \multirow{2}{*}{$\begin{array}{l}p \text {-value } \\
<0.001\end{array}$} & \multirow[t]{2}{*}{ OR } & \multicolumn{3}{|c|}{$95 \% \mathrm{Cl}$} & \multirow[t]{2}{*}{ p-value } \\
\hline & & 1.010 & - & 1.026 & & & & & & \\
\hline HDL & 0.834 & 0.743 & - & 0.937 & 0.002 & & & & & \\
\hline MHR & 2.792 & 1.663 & - & 4.690 & $<0.001$ & 2.792 & 1.663 & - & 4.690 & $<0.001$ \\
\hline
\end{tabular}

Biochemical parameters were measured using the Beucher Coulter AU5800 (Beckman Coulter GmbH Europark Fichtenhain B 1347807 Krefeld, Germany) automatic measuring device.

Mean, standard deviation, median, minimum, maximum, frequency, and percentage values were used as descriptive statistics. The distribution of variables was checked using a Shapiro-Wilk test. An independent samples t-test and a MannWhitney U-test were used to compare the quantitative data, and a Chi-square Fisher's exact test was used to compare the qualitative data. A ROC analysis and logistic regression were used to determine the effect levels. SPSS 26.0 was used for statistical analysis.

\section{RESULTS}

Of the 81 patients included in the present study, $48.1 \%(n=$ 39) were males and $51.9 \%(n=42)$ were females. The mean age of the patients was $69.9 \pm 10.6$ years. The mean and standard deviation values of the vital signs and hematological 
and biochemical parameters are shown in Table I. The age and gender distributions of the patients with and without mortality did not differ significantly $(p>0.05)$.

Table IV: The area under the curve for monocyte, HDL and MHR.

\begin{tabular}{|l|l|l|l|l|l|}
\hline & Area under curve & \multicolumn{3}{l|}{$\%$ 95 Cl } & p-value \\
\hline Monocyte & 0.939 & 0.890 & - & 0.987 & $<\mathbf{0 . 0 0 1}$ \\
\hline HDL & 0.271 & 0.149 & - & 0.393 & $<\mathbf{0 . 0 0 1}$ \\
\hline MHR & 0.965 & 0.930 & - & 1.000 & $<0.001$ \\
\hline
\end{tabular}

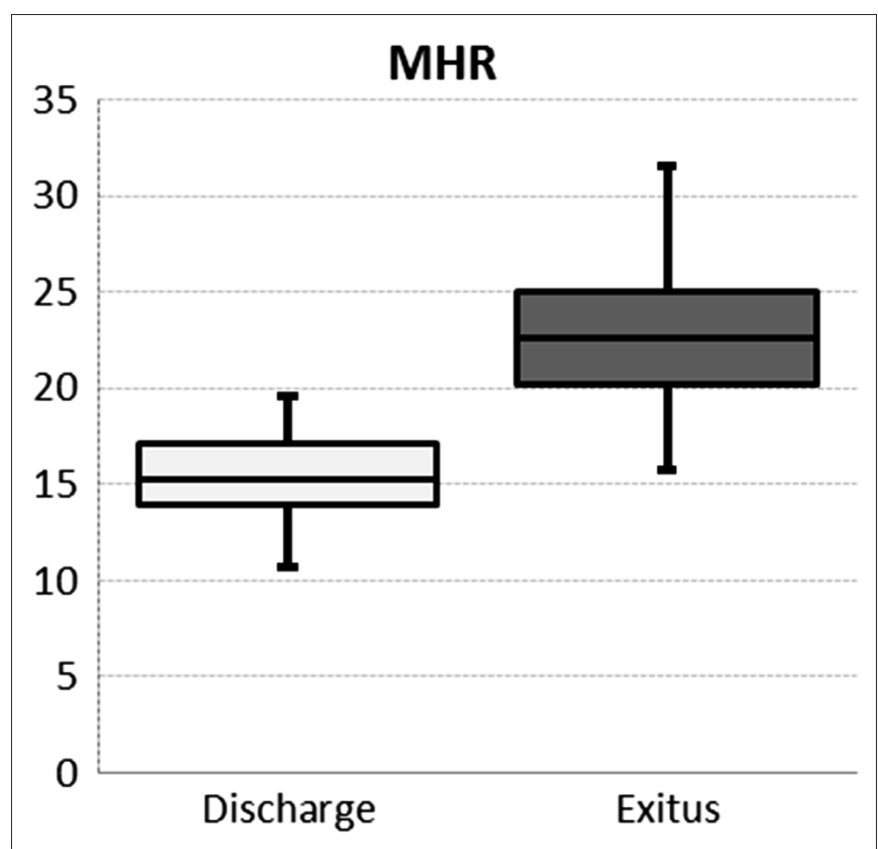

Figure 1: MHR distribution of groups.

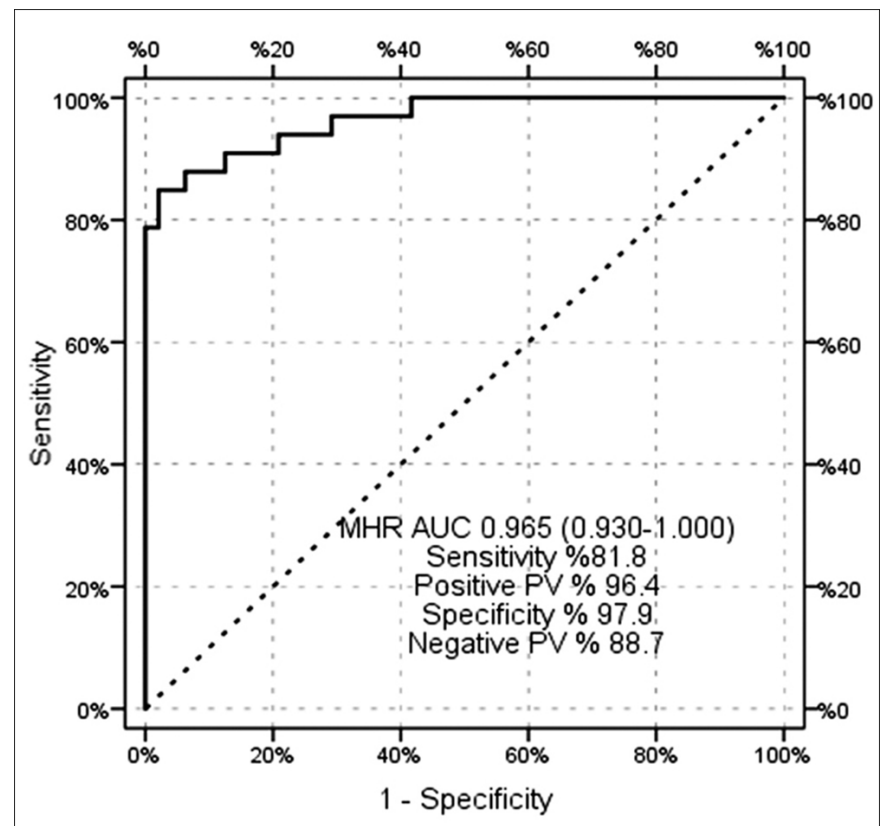

Figure 2: ROC analysis for MHR.
Further, these groups did not differ significantly $(p>0.05)$ in terms of mortality, fever, heart rate, systolic or diastolic blood pressure, glucose, urea, creatinine, ALT, AST, sodium, potassium, calcium, albumin, hemoglobin, hematocrit, or platelet values. However, the numbers of monocytes and MHRs were significantly higher in the group with mortality than in the group without mortality $(p<0.05)$. On the other hand, HDL values were significantly lower in the mortality group than in the non-mortality group ( $p<0.05$; Figure 1 , Table II).

Significant efficacy $(p<0.05)$ of the monocyte, HDL, and MHR values was observed in the univariate model. Significant independent efficacy $(p<0.05)$ of the MHR was observed in the multivariate regression model (Table III). Significant efficacy $(p<0.001)$ of the monocyte value $[0.939(0.890-0.987)]$, the HDL value $[0.271(0.149$ $0.393)]$, and the MHR value [0.965 (0.930 - 1.00)] was observed in the prediction of mortality (Table IV, Figure 2). The sensitivity of the MHR cutoff value of 19 was $81.8 \%$, the positive predictive value was $96.4 \%$, the specificity value was $97.9 \%$, and the negative predictive value was $88.7 \%$.

\section{DISCUSSION}

The main findings of this study were that patients diagnosed with mesenteric embolism had significantly higher monocyet counts, lower HDL cholesterol levels, and higher MHR. To the best of authors' knowledge, these findings are the first study in the literature to show an increase in MHR in patients with mesenteric. Increasing of monocyte counts and decreasing of $\mathrm{HDL}$ cholesterol levels were another important findings of this study.

The most important causes of high mortality in acute mesenteric embolism include difficulties and delays in diagnosis before necrosis occurs in the intestinal tissue. Therefore, the recognition of this disease without evidence of necrosis or peritoneal inflammation in the intestine is one of the important factors affecting mortality. ${ }^{13-15}$ Biochemical and hematological markers can be applied simply, which are inexpensive, and provide rapid results. However, data regarding the use of these markers to predict mortality in cases of mesenteric embolism are limited. To the best of authors' knowledge, no previous study has investigated the effectiveness of MHR in predicting mortality in cases of mesenteric embolism, which are considered to result from a thromboembolic cause. Therefore, this study appears to be the first in the literature to reveal that MHR as an independent marker of mortality in patients diagnosed with mesenteric embolism.

In the present study, monocyte counts were significantly higher in the mortality group than in the non-mortality group. Monocytes play an important role in the progression of atherosclerosis as an immune-mediated process by binding to 
adhesion molecules expressed on the damaged vascular endothelium. 9,16,17 The role of monocytes in atherosclerosis is not limited to macrophage function in the arterial wall. Further, immune-stimulating agents can affect growth factors, oxidised lipids, platelet-induced activation products, and many soluble factors associated with atherosclerosis, such as eicosanoids. As a result, monocytes play a role in the pathogenesis of vascular diseases and in complications that can occur through the inflammatory process. ${ }^{18}$ The data obtained in the present study revealed that an increased monocyte count is an important parameter in mortality prediction.

HDL consists of subgroups of different diameters, densities, and lipid and protein compositions. Several of the biological activities of HDL result from its heterogeneous structure. Aside from reverse cholesterol transport, HDL has demonstrated antioxidant, anti-inflammatory, antiapoptotic, anti-thrombotic, and antiatherosclerotic effects., ${ }^{9,19,20} \mathrm{HDL}$ prevents monocyte flow into the arterial wall with reduced $\mathrm{F}$ actin, thereby reducing the expression of CDI1b on monocytes and endothelial adhesion molecules and preventing monocytes from adhering to the endothelium. ${ }^{21}$ These processes protect endothelial cells from inflammation and oxidative stress. ${ }^{2,4}$ Reduction of HDL cholesterol in the blood would reverse these processes and eliminate this shield of protection against inflammation and oxidative processes. In the present study, HDL was shown to be significantly low in patients with mesenteric embolism resulting in death. Therefore, a decrease in HDL cholesterol levels may serve as an effective marker for mortality prediction, as it would render monocytes insufficient to neutralize pro-inflammatory and pro-oxidant effects.

In the present study, the cutoff value of MHR was above 19, its sensitivity was $81.5 \%$, its specificity was $97.9 \%$, its positive predictive value was $96.4 \%$, and its negative predictive value was $88.7 \%$. This study is the first to demonstrate, through multivariate analysis, that MHR is a very strong independent predictor of mortality in patients diagnosed with mesenteric embolism. High monocyte counts and low HDL cholesterol levels have been associated with inflammation and oxidative stress, ${ }^{2-5}$ and MHR has been highlighted as a new prognosis marker for some cardiovascular diseases. ${ }^{6-8}$ The literature has also emphasised that the MHR has an independent predictive role in revealing the relationships between cardiovascular diseases (e.g., ST elevation myocardial infarction, saphenous vein graft disease, and primary hypertension resulting in asymptomatic organ damage), carotid artery intima thickness, the left ventricular mass index, urinary protein and albumin levels, and metabolic syndrome. ${ }^{10,11,22,23}$ However, no previous study has revealed the relationship between the MHR and mortality in cases of mesenteric embolism.

There are some limitations in this study. One of them is the retrospective design of the study, and also the number of patients included in the study. In this study, the number of patients was limited to 81 . Prospective studies involving more patients may provide more meaningful information.

\section{CONCLUSION}

The mortality rate of acute mesenteric embolism is affected by early diagnosis and early revascularisation treatments. The use of predictors for the prediction of mortality is very important for these patients in order to apply treatment modalities faster. MHR value can be used as a strong predictor. The cutoff values obtained may contribute to the literature, and to large-scale studies in which multi-centred, prospective patients will be analysed.

\section{ETHICAL APPROVAL:}

The study was approved by Ethics Committee of NonInterventional Clinical Researches of Adana City Research and Training Hospital (Decision No.65 and Decision Date 18.12.2019).

\section{PATIENTS' CONSENT:}

The data was collected retrospectively from Hospital Automation System and Archives, after obtaining necessary permission from the management.

\section{CONFLICT OF INTEREST:}

Authors declared no conflict of interest.

\section{AUTHORS' CONTRIBUTION:}

BSA, AK: Made substantial contributions to the conception or design, acquisition, analysis, or interpretation of data for the work, drafted the work and revised it critically for important intellectual content; approved the final version to be published; agreed to be accountable for all aspects of the work in ensuring that questions related to the accuracy or integrity of any part of the work are appropriately investigated and resolved. Planned and designed the work; analysed, or interpreted data for the work; drafted the work or revised it critically for important intellectual content. Drafted the work and revised it critically for important intellectual content.

\section{REFERENCES}

1. Kassahun WT, Schulz T, Richter O, Hauss J. Unchanged high mortality rates from acute occlusive intestinal ischemia: Six year review. Langenbecks Arch Surg 2008; 393(2):163-71.

2. Açıkgöz SK, Açıkgöz E, Şensoy B, Topal S, Aydoğdu S. Monocyte to high-density lipoprotein cholesterol ratio is predictive of in-hospital and five-year mortality in STsegment elevation myocardial infarction. Cardiol J 2016; 23 (5):505-12.

3. Bolayir A, Gokce SF, Cigdem B, Bolayir HA, Yildiz OK, Bolayir $\mathrm{E}$, et al. Monocyte/high-density lipoprotein ratio predicts the mortality in ischemic stroke patients. Neurol NeurochirPol 2018; 52(2):150-5. 
4. Kundi $H$, Gok M, Kiziltunc $E$, Cetin M, Cicekcioglu $H$, Cetin $Z G$, et al. Relation between monocyte to high-density lipoprotein cholesterol ratio with presence and severity of isolated coronary artery ectasia. Am J Cardiol 2015; 116 (11):1685-9.

5. Kadihasanoglu M, Karabay E, Yucetas U, Erkan E, Ozbek E. Relation between monocyte to high-density lipoprotein cholesterol ratio with presence and severity of erectile dysfunction. Aktuelle Urol 2018;49(3): 256-61.

6. Karataş MB, Çanga Y, Özcan KS, Ipek G, Güngör B, Onuk T, et al. Monocyte to high-density lipoprotein ratio as a new prognostic marker in patients with STEMI undergoing primary percutaneous coronary intervention. Am J Emer Med 2016; 34(2):240-4.

7. Cetin MS, Ozcan CEH, Kalender E, Aydin S, Topaloglu S, Kisacik HL, et al. Monocyte to HDL cholesterol ratio predicts coronary artery disease severity and future major cardiovascular adverse events in acute coronary syndrome. Heart Lung and Circulation 2016; 25(11):1077-86.

8. You S, Zhong C, Zheng D, Xu J, Zhang X, Liu H, et al. Monocyte to HDL cholesterol ratio is associated with discharge and 3-month outcome in patients with acute intracerebral hemorrhage. J Neurol Sci 2017; 15(372): 157-61.

9. Tani S, Matsumoto M, Anazawa T, Kawamata H, Furuya S, Takahashi $\mathrm{H}$, et al. Development of a model for prediction of coronary atherosclerotic regression: Evaluation of highdensity lipoprotein cho esterol level and peripheral blood monocyte count. Heart and Vessels 2012; 27(2):143-50.

10. Akboga MK, Yayla C, Balci K, Ozeke O, Maden O, Kisacik H, et al. Relationship between serum albumin level and monocyte-to-high-density lipoprotein cholesterol ratio with saphenous vein graft disease in coronary bypass. Thoracic Cardio Surg 2017; 65(4):315-21.

11. Aydin E, Ates I, Fettah AM, Yilmaz N, Dede F. The ratio of monocyte frequency to HDL cholesterol level as a predictor of asymptomatic organ damage in patients with primary hypertension. Hyper Res 2017; 40(8):758-64.

12. Tilsed JVT, Casamassima A, Kurihara H, Mariani D, Martinez I, Pereira J, et al. ESTES guidelines: Acute mesenteric ischaemia. Eur J Trauma Emerg Surg 2016; 42(2):253-70.

13. Boley SJ, Feinstein FR, Sammartano R, Brandt LJ, Sprayre- gen S. New concepts in the management of emboli of the superior mesenteric artery. Surg Gynecol Obstet 1981; 153(4):561-9.

14. Lobo-Martinez E, Merono CE, Sacco O, Martinez ME. Embolectomy in mesenteric ischemia. Rev Esp Enferm Dig 1993; 83(5):351-4.

15. Hot S, Duraker N, Sarı A, Çetin K. The value of d-dimer in diagnosis of acute mesenteric Ischemia and differential diagnosis from acute pancreatitis and acute cholecystitis. Dicle Med J 2016; 43(1):88-92.

16. Ammirati E, Moroni F, Magnoni M, Di Terlizzi S, Villa C, Sizzano F, et al. Circulating CD14+ and CD14 high CD16classical monocytes are reduced in patients with signs of plaque neovascularization in the carotid artery. Atherosclerosis 2016; 255:171-8.

17. Aukrust $P$, Halvorsen $B$, Yndestad A, Ueland $T$, Oie E, Otterdal $\mathrm{K}$, et al. Chemokines and cardiovascular risk. Arteriosclerosis Throm Vascul Biol 2008; 28(11):1909-19.

18. Gratchev A, Sobenin I, Orekhov A, Kzhyshkowska J. Monocytes as a diagnostic marker of cardiovascular diseases. Immunobiology 2012; 217(5):476-82.

19. Ganjali S, Momtazi AA, Banach M, Kovanen PT, Stein EA, Sahebkar A. HDL abnormalities in familial hypercholesterolemia: Focus on biological functions. Prog Lipid Res 2017; 67:16-26.

20. Gomaraschi M, Basilico N, Sisto F, Taramelli D, Eligini S, Colli $\mathrm{S}$, et al. High-density lipoproteins attenuate interleukin-6 production in endothelial cells exposed to pro-inflammatory stimuli. Biochim Biophys Acta 2005; 1736(2): 136-43.

21. Murphy AJ, Woollard KJ, Hoang A, Mukhamedova N, Stirzaker RA, McCormick S, et al. High-density lipoprotein reduces the human monocyte inflammatory response. Arteriosclerosis Throm Vascu Biol 2008; 28(11):2071-7.

22. Yilmaz S, Akboga MK, Sen F, Balcı KG, Aras D, Temizhan A, et al. Usefulness of the monocyte-to-high-density lipoprotein cholesterol ratio to predict bare metal stent restenosis. Bio Med 2016; 10(9):959-66.

23. Mottillo S, Filion KB, Genest J, Joseph L, Pilote L, Poirie P, et al. The metabolic syndrome and cardiovascular risk: A systematic review and meta-analysis. J Am Coll Cardiol 2010; 56(14):1113-32. 